\title{
MiR-279-3p regulates deltamethrin resistance through CYP325BB1 in Culex pipiens pallens
}

Xixi Li ${ }^{1,2+}$, Shengli Hu ${ }^{1,3+}$, Hongbo Zhang ${ }^{1}$, Haitao Yin ${ }^{1}$, Huan Wang ${ }^{1}$, Dan Zhou' ${ }^{1}$, Yan Sun ${ }^{1}$, Lei Ma', Bo Shen $^{1 *}$ and Changliang Zhu ${ }^{1}$

\begin{abstract}
Background: The overuse of insecticides to control insect vectors has promoted extensive insecticide resistance in mosquitoes. In this study, the functions of microRNA (miR)-279-3p and its target CYP325BB1 in the regulation of deltamethrin resistance in Culex pipiens pallens was investigated.
\end{abstract}

Methods: Quantitative real-time reverse transcription PCR was used to detect the expression levels of miR-279-3p and CYP325BB1. Then, the dual-luciferase reporter assay system, RNA interference, CDC bottle bioassay and Cell Counting Kit-8 (CCK-8) assay were used to explore the roles of these molecules in deltamethrin resistance both in vivo and in vitro.

Results: The expression patterns of miR-279-3p and CYP325BB1 were compared between deltamethrin-sensitive (DS-strain) and deltamethrin-resistant (DR-strain) mosquitoes. Luciferase activity was downregulated by miR-279-3p, the effect of which was ablated by a mutation of the putative binding site for CYP325BB1. In DR-strain mosquitoes, the expression of miR-279-3p was increased by microinjection and oral feeding of miR-279-3p agomir (mimic). CYP325BB1 mRNA levels were downregulated, which resulted in a higher mortality of the mosquitoes in miR-279-3p mimictreated groups. In the DS-strain mosquitoes, microinjection of a miR-279-3p inhibitor decreased miR-279-3p expression, whereas the expression of CYP325BB1 was increased; the mortality of these mosquitoes decreased significantly. In addition, overexpression of pIB/N5-His-CYP325BB1 changed the sensitivity of C6/36 cells to deltamethrin in vitro. Also in DR-strain mosquitoes, downregulation of CYP325BB1 expression by microinjection of si-CYP325BB1 increased mosquito mortality in vivo.

Conclusions: These findings provide empirical evidence of the involvement of miRNAs in the regulation of insecticide resistance and indicate that miR-279-3p suppresses the expression of CYP325BB1, which in turn decreases deltamethrin resistance, resulting in increased mosquito mortality. Taken together, the results provide important information for use in the development of future mosquito control strategies.

Keywords: MicroRNA, Cytochrome P450, Insecticide resistance, Mosquito

*Correspondence: shenbo@njmu.edu.cn

${ }^{+}$Xixi Li and Shengli Hu contributed equally to this work

${ }^{1}$ Department of Pathogen Biology, Nanjing Medical University, Nanjing, Jiangsu 211166, People's Republic of China

Full list of author information is available at the end of the article

\section{Background}

Mosquitoes are insect vectors of pathogens, including those causing serious human diseases, such as filariasis, encephalitis, West Nile fever, dengue fever and malaria, which threaten public health worldwide [1, 2]. Annually, insect-borne diseases cause illnesses in excess of half original author(s) and the source, provide a link to the Creative Commons licence, and indicate if changes were made. The images or other third party material in this article are included in the article's Creative Commons licence, unless indicated otherwise in a credit line to the material. If material is not included in the article's Creative Commons licence and your intended use is not permitted by statutory regulation or exceeds the permitted use, you will need to obtain permission directly from the copyright holder. To view a copy of this licence, visit http://creativecommons.org/licenses/by/4.0/. The Creative Commons Public Domain Dedication waiver (http://creativeco mmons.org/publicdomain/zero/1.0/) applies to the data made available in this article, unless otherwise stated in a credit line to the data. 
a billion people, of whom about 1 million die, including more than 400,000 children, one of whom dies from malaria every other minute of every day [3, 4]. Mosquitoborne diseases pose a growing threat to human health. Currently, the control of mosquito vectors is still an effective means to prevent and control mosquito-borne diseases. However, in recent decades, extensive insecticide use to control insect vectors has led to insecticide resistance, which is the main obstacle to mosquito control $[5,6]$. Numerous studies have shown that increased metabolic detoxification of insecticides and decreased sensitivity of the target have resulted in complex multiple insecticide resistance [7]. Cytochrome P450 monooxygenases (P450s) have been shown to function in metabolic resistance by increasing the expression or activity of detoxification genes in mosquitoes [8]. Therefore, acquiring a detailed understanding of the molecular biology underlying insecticide resistance, particularly the posttranscriptional regulation of metabolic detoxification (especially for the P450s family), is important to develop novel approaches for pathogen and vector control [9].

MicroRNAs (miRNAs), which are noncoding RNAs of 22-23 nt, promote transcript decay and repress messenger RNA (mRNA) translation [10]. They bind to imperfect complementary sequences in the $3^{\prime}$ untranslated regions ( $3^{\prime}$-UTRs) of their target mRNAs [11]. In mosquito species, their particular developmental processes are affected by lineage-specific miRNAs, which might be developed as targets for vector control [12]. MicroRNAs interact with multiple target genes to elicit biological functions, and aberrant miRNA expression has been observed in development, metabolism, host-pathogen interactions and insecticide resistance. For example, in Anopheles, miR-276 affects the mosquito reproductive cycle and Plasmodium falciparum development via metabolic balancing [13]. The characterization and insecticide resistance functions of several mosquito miRNAs have recently been clarified [14-17]; however, the roles of mosquito-specific miRNAs and their participation in specific insecticide resistance events remain mostly unknown [18]. In insecticide-resistant mosquitoes, several miRNAs are dysregulated, suggesting that they may participate in insecticide resistance events. Our previous report on the high-throughput sequencing of the mosquito Culex pipiens pallens identified miR-279-3p as being highly expressed in deltamethrin-sensitive (DS-strain) mosquitoes compared deltamethrin-resistant (DR-strain) mosquitoes [19]; however, its role has remained undetermined.

The aim of the present study was to investigate the regulatory mechanism of deltamethrin resistance by determining the function of the miR-279-3p in the regulation of its target, CYP325BB1 (cytochrome P450 325bb1).
By analyzing the expression patterns of miR-279-3p and CYP325BB1 in DR- and DS-strain mosquitoes subjected to RNA interference (RNAi), and function detection using $\mathrm{CDC}$ bottle bioassays, we detected a negative correlation between miR-279-3p and CYP325BB1 with insecticide resistance in vivo. Cell viability analysis using the Cell Counting Kit-8 (CCK-8) assay validated the result in vitro. Thus, our data suggest that miR279-3p might affect deltamethrin resistance by regulating CYP325BB1 expression in Cx. pipiens pallens. These findings have important implications for understanding the mechanism of insecticide resistance and for developing mosquito control strategies in the future.

\section{Methods}

\section{Mosquito strains and cell lines}

Two strains of $C x$. pipiens pallens with different resistance levels to deltamethrin were used in this study. The DS-strain of Cx. pipiens pallens used in this study was obtained from Ji Nan University and subsequently reared in our laboratory at $28{ }^{\circ} \mathrm{C}, 70-80 \%$ relative humidity, 14:10 h light/dark cycle. The DS-strain mosquitoes had not been exposed to any insecticides. The DR-strain was selected from the DS-strain by constant exposure to deltamethrin (at lethal concentration $50\left[\mathrm{LC}_{50}\right]$; concentration which kills $50 \%$ of sample population) and was screened over 80 generations. There were 4000 larvae screened for each pool (three pools/generation). The $\mathrm{LC}_{50}$ of the DS- and DR-strains was 0.05 and $8.5 \mathrm{mg} / \mathrm{l}$, respectively. The resistance ratio of $\mathrm{LC}_{50}\left(\mathrm{RR}_{50}\right)$ of the DR-strain was 170. Deltamethrin (technical grade, 99.0\%) was obtained from Jiangsu Provincial Center for Disease Control and Prevention (Jiangsu, China). Larvae were grown in dechlorinated tap water and fed with fish food powder (Tetramin; Tetra, Pirmasens, Germany) every 2 days. Adult mosquitoes were maintained in cages with constant access to a 5\% glucose solution. Female mosquitoes were fed on mouse blood to reproduce the next generation every 3-4 weeks. Procedures for blood-feeding with mice in our laboratory were approved by The National Science and Technology of China and People's Government of Jiangsu Province Animal Care and Use Committee and Institutional Review Board (No. IACUC-1812047).

The HEK293T cell line was grown in complete high glucose Dulbecco's modified Eagle's medium (DMEM; Gibco, Grand Island, NY, USA) containing 10\% (v/v) fetal bovine serum (FBS; Gibco) and $100 \mathrm{U} / \mathrm{ml}$ penicillin-streptomycin solution (Gibco) at $37{ }^{\circ} \mathrm{C}$ under $5 \%$ $\mathrm{CO}_{2}$. Cells $\left(6 \times 10^{4} /\right.$ well $)$ were seeded and incubated in $2.5 \mathrm{ml}$ of complete growth medium in a 6-well plate for $24 \mathrm{~h}$ until they achieved $>80 \%$ confluency. The mosquito C6/36 cell line (CRL-1660; ATCC, Manassas, VA, USA) 
was cultured in DMEM supplemented with $10 \%$ FBS. The cells were plated in a 6-well plate and were grown in a $5 \%$ $\mathrm{CO}_{2}$-humidified incubator at $28{ }^{\circ} \mathrm{C}$. Cells $\left(5 \times 10^{5} /\right.$ well $)$ were plated and incubated in $2.5 \mathrm{ml}$ of complete growth medium in a 6-well plate for $24 \mathrm{~h}$, until they achieved the required density of $60-80 \%$ for transfection. All cells were confirmed to be negative for mycoplasma contamination regularly.

\section{RNA and genomic DNA extraction}

The RNAiso Plus reagent (Takara, Dalian, China) was used to extract total RNA from the female adult mosquitoes $(n=5)$ of the DS- and DR-strains at 3 days postemergence (3 days PE). Genomic DNA (gDNA) was extracted from 3-day PE female adult mosquitoes using a MiniBEST Universal Genomic DNA Extraction Kit Ver. 5.0 (Takara) according to the manufacturer's protocol. The quality and quantity of the RNA and gDNA were checked using a Thermo Scientific ${ }^{\mathrm{TM}}$ NanoDrop 2000 instrument (Thermo Fisher Scientific, Waltham, MA, USA).

\section{Quantitative real-time reverse transcription PCR analyses}

A PrimeScript RT Reagent Kit (Takara) and PrimeScript ${ }^{\mathrm{TM}}$ RT Master Mix (Takara) were used to synthesize complementary DNA (cDNA) from $1 \mu \mathrm{g}$ of total RNA following the manufacturer's instructions. Then, $4 \mu \mathrm{l}$ of 1:10 diluted cDNA solution was used as the template for quantitative real-time PCR (qPCR), which was performed using the Power SYBR Green PCR Master Mix (Applied Biosystems, Foster City, CA, USA). The PCR reaction mix $(20 \mu \mathrm{l})$ contained forward and reverse PCR primers (10 pmol) for miR-279-3p, U6, CYP325BB1 and $\beta$-actin, designed using Primer Premier 6.0 software (Premier Biosoft, Palo Alto, CA, USA; Table 1). Stem-loop reverse transcription (RT)-PCR was used to measure the expression of miR-279-3p [20]. The PCR cycling conditions were: $50{ }^{\circ} \mathrm{C}$ for $2 \mathrm{~min}$; then $95^{\circ} \mathrm{C}$ for $10 \mathrm{~min}$; followed by 40 cycles of $95{ }^{\circ} \mathrm{C}$ for $15 \mathrm{~s}$ and $60{ }^{\circ} \mathrm{C}$ for $1 \mathrm{~min}$; meltingcurve analysis was then performed on an ABI Prism 7300 real-time PCR Instrument (Applied Biosystems). The miR-279-3p relative expression level was normalized to that of the internal control small nuclear RNA (U6), which is standard for miRNA expression normalization [21], and the expression level of CYP325BB1 was normalized to that of $\beta$-actin from the DS- and DR-strains [22]. The DS-strain expression level was designated as 1 , and the negative control (NC) was used to compare the gene expression levels. Each experiment used RNA from three biological replicates, and each cDNA sample was PCR amplified in triplicate. The $2^{-\Delta \Delta \mathrm{Ct}}$ method was used to calculate the expression levels [23].

\section{Identifying the potential target of miR-279-3p}

To identify the putative gene targets of miR-279-3p, we used 3'-UTR sequences from the Culex quinquefasciatus genome in the RNAhybrid target prediction program [24]. We focused on the cytochrome P450 family of genes $(C Y P)$ that participate in the regulation of insecticidal resistance of mosquitoes, and only CYP325BB1 was identified as a potential target of miR-279-3p. To assess the conservation of the $3^{\prime}$-UTR, we amplified the $3^{\prime}$-UTR from $C x$. pipiens pallens. The $3^{\prime}$-UTR sequence of CYP325BB1 in Cx. pipiens pallens was $100 \%$ identical with that from $C x$. quinquefasciatus.

\section{PMIR-REPORT vector construction and dual-luciferase reporter assay}

To amplify the wild-type $3^{\prime}$-UTR (3'-UTR-WT) and the mutated $3^{\prime}$-UTR (3'-UTR- $\triangle$ ) of CYP325BB1 of $C x$. pipiens pallens, primers for CYP325BB1 $3^{\prime}$-UTR-WT/A (Table 1) were designed based on $C x$. quinquefasciatus transcripts, which amplified the 3 '-UTR region containing the miR-279-3p complementary sequences. Mutagenesis of the $3^{\prime}$-UTR (CTAGTCA) comprised replacing the WT binding site with CGACTGA. The Cx. pipiens pallens CYP325BB1 3'-UTR-WT and 3'-UTR- $\Delta$ sequences containing the putative seed region of the miR-279-3p binding sites were amplified and sequenced using the T/A cloning method. PCR products were run in $2.0 \%$

Table 1 Primers used for PCRs and vector constructions

\begin{tabular}{lll}
\hline Name of primer & Forward $\left(5^{\prime}-3^{\prime}\right)$ & Reverse $\left(5^{\prime}-3^{\prime}\right)$ \\
\hline miR-279-3p (qRT-PCR) & ACACTCCAGCTGGGTGACTAGATCCACAC & TGGTGTCGTGGAGTCG \\
U6 & GCTTCGGCTGGACATATACTAAAAT & GAACGCTTCACGATTTTGCG \\
CYP325BB1 & TGCTGACCAGCGAACGAA & AGACCACCTTTCACCATCCC \\
B-actin & AGCGTGAACTGACGGCTCTTG & ACTCGTCGTACTCCTGCTTGG \\
CYP325BB1 3'-UTR-WT & TATCGGCTGTGGACTGACCTT & AAACCCATTTGGCATAAGACG \\
CYP325BB1 3'-UTR- $\triangle$ & TATCGGCTGTGGACTGACCTT & CCAAGCTTTTTTTGCCCACCAAGGTTT \\
PIBN5-His-CYP323BB1 & GGACTAGTGAGATGGAATGCTGTTCGAAGTGCTCC & CCGCTCGAGCGTTTATTTCTCTTAGTCAACCAAACT \\
\hline
\end{tabular}


agarose gels and purified using a MiniBEST Agarose Gel DNA Extraction Kit Ver. 4.0 (Takara), and then cloned into vector pMD 19-T (Takara). The resultant plasmid was transferred into Escherichia coli One Shot ${ }^{\circledR}$ TOP10 Competent Cells (Invitrogen, Thermo Fisher Scientific, Carlsbad, CA, USA). The UTR sequences were then ligated into the HindIII and XbaI sites, which are located downstream of the Renilla translational stop codon in the pMIR-REPORT miRNA Expression Reporter Vector (Promega, Madison, WI, USA), to create the luciferase constructs. The pMIR-UTR-WT (6 ng) or pMIR-UTR- $\Delta$ were treated with miR-279-3p mimic $(6 \mu$ l; GenePharma, Shanghai, China) and NC (GenePharma) along with PGL4.7 (6 ng; Promega), which were cotransfected using the FuGENE HD transfection reagent (Promega) into HEK293T cells (> 80\% confluency). The Dual-Luciferase ${ }^{\circledR}$ Reporter Assay System (Promega) was then used for the reporter assay at $48 \mathrm{~h}$ after transfection. PGL4.7 provided the constitutive Firefly luciferase expression and was cotransfected as an internal control. Renilla luciferase was normalized to Firefly luciferase expression in each sample. The luciferase activity was detected at 560 and $480 \mathrm{~nm}$ using an M200 microplate fluorescence reader (Tecan, Lyon, France). The transfections were performed three times, and treatment was performed in triplicate.

\section{Microinjection}

For the microinjection of miRNA, female adult mosquitoes were collected within $12 \mathrm{~h} \mathrm{PE}$ and frozen at $-20{ }^{\circ} \mathrm{C}$ for $3-5 \mathrm{~min}$. These mosquitoes were divided into three groups and prepared for injection through the thorax using a Nanoject III instrument (Drummond, Broomall, PA, USA). The miR-279-3p mimic or $\mathrm{NC}(0.5 \mu \mathrm{l} ; 20 \mathrm{nmol} / \mathrm{l})$ were injected into the DR-strain mosquitoes (miR-279-3p mimic/NC group) under the same conditions. The miR-279-3p inhibitor or NC $1(\mathrm{NC} 1,0.5 \mu \mathrm{l} ; 20 \mathrm{nmol} / \mathrm{l})$ was injected into the DSstrain mosquitoes (miR-279-3p inhibitor/NC1). For the microinjection of small interfering RNA (siRNA), 12-h PE DR-strain mosquitoes from the experimental group (si-CYP325BB1) and NC group were injected with $69 \mathrm{nl}$ of si-CYP325BB1 or NC $(5 \mu \mathrm{g} / \mu \mathrm{l})$ under the same conditions. Thereafter, the mosquitoes were maintained with a constant light/dark cycle $(14 / 10 \mathrm{~h})$ at $28{ }^{\circ} \mathrm{C}$ and $70-80 \%$ humidity. After $72 \mathrm{~h}$, the expression levels of miR-279-3p and CYP325BB1 were validated using qRTPCR. Three biological replicates (each comprising 15 female mosquitoes) with three technical replicates were performed. GenePharma designed and produced all the injected RNA products (Table 2).

\section{Oral feeding}

For the oral feeding experiments, pupae of the DR-strain were collected and placed in three cages until eclosion, and then fasted for $12 \mathrm{~h}$. The mosquitoes of the control group were fed with $5 \%(\mathrm{w} / \mathrm{v})$ glucose on a sponge wick ( $3 \mathrm{M}$, Minneapolis, MN, USA), while the NC group and the experimental group (miR-279-3p mimic) were given $\mathrm{NC}$ and miR-279-3p mimic (100 nmol/l) dissolved in 5\% $(\mathrm{w} / \mathrm{v})$ glucose dissolved in DEPC-treated water, respectively. After $48 \mathrm{~h}$ of treatment, the RNAs of female adult mosquitoes were extracted to validate the expression levels of miR-279-3p and CYP325BB1. The miR-279-3p mimic and $\mathrm{NC}$ were obtained from GenePharma.

\section{U.S. Centers for Disease Control and Prevention bottle bioassay}

To detect the mosquitoes' sensitivity to deltamethrin after alteration of the miR-279-3p and CYP325BB1 levels, U.S. Centers for Disease Control and Prevention (CDC) bottle bioassays were conducted according to published guidelines (https://www.cdc.gov/malaria/ resources/pdf/fsp/ir_manual/ir_cdc_bioassay_en.pdf) [25]. Each bottle $(250 \mathrm{ml})$ and its cap were rolled and inverted to coat them with $1 \mathrm{ml}$ deltamethrin solution (7 mg/l for DR-strain; $0.01 \mathrm{mg} / \mathrm{l}$ for DS-strain). In parallel, a control bottle was coated with $1 \mathrm{ml}$ of acetone, and then all bottles were left to dry in the dark. Mosquitoes from the designated groups (20/bottle) were exposed to deltamethrin or acetone for $2 \mathrm{~h}$. Following exposure, the mosquitoes were monitored at 15 -min intervals for $2 \mathrm{~h}$ and the percentage mortality ( $y$-axis) was plotted against time ( $x$-axis) using a linear scale.

Table 2 Sequences of the miR-279-3p mimic, negative control, miR-279-3p inhibitor, inhibitor negative control 1 and si-CYP325BB1

\begin{tabular}{lll}
\hline Name & Sense $\left(5^{\prime}-3^{\prime}\right)$ & Antisense $\left(5^{\prime}-3^{\prime}\right)$ \\
\hline miR-279-3p mimic & UGACUAGAUCCACACUCAUUA & AUGAGUGUGGAUCUAGUCAUU \\
Negative control (NC) & UUCUCCGACGUGUCACGUTT & ACGUGACACGUUCGGAGAATT \\
miR-279-3p inhibitor & UAAUGAGUGUGGAUCUAGUCAC & \\
Inhibitor NC 1 (NC1) & CAGUACUUUUGUGUAGUACAA & UUUAAGAGCACUUCCAGCCTT \\
si-CYP325BB1 & GGCUGGAAGUGCUCUUAAATT
\end{tabular}


PIB/V5-His vector construction, transfection and detection Standard molecular biology procedures were used for plasmid constructions. Standard overlap PCR was performed to amplify the open reading frame (ORF) of $C Y$ P325BB1 using the pIB/V5-His-CYP325BB1 primer pair (Table 1) from Cx. quinquefasciatus, which was ligated between unique restriction enzyme sites (SpeI/ XhoI) of the eukaryotic expression vector pIB/V5-His. The positive recombinant plasmid was named $\mathrm{pIB} /$ V5-His-CYP325BB1 and was confirmed using DNA sequencing.

C6/36 cells at $60-80 \%$ confluence were used for transfection. Plasmid DNA (pIB/V5-His-CYP325BB1) was diluted in complete growth medium to $1.5 \mathrm{ng} / 100$ $\mu \mathrm{l}$ and $5 \mu \mathrm{l}$ of FuGENE HD transfection reagent was added, followed by incubaion at room temperature with shaking for $25 \mathrm{~min}$. pIB/V5-His was transfected as a control. Three biological replicates with three technical replicates were performed.

To evaluate the transfection efficiency of CYP325BB1, after $48 \mathrm{~h}$ of transfection, the mRNA and protein levels of CYP325BB1 in transiently transfected C6/36 cells were detected. Total RNA was isolated from the transfected cells and subjected to qRT-PCR to check the expression level of CYP325BB1. Protein was extracted from transfected cells, followed by washing in phosphate buffered saline (PBS), digestion in trypsin solution and lysis using radioimmunoprecipitation assay (RIPA) buffer (Beyotime, Jiangsu, China). The protein concentration was tested using a bicinchoninic acid (BCA) Protein Assay kit (Pierce, Rockford, IL, USA). Soluble protein $(50 \mu \mathrm{g})$ was denatured and subjected to $10 \%$ sodium dodecyl sulfate-polyacrylamide gel electrophoresis. The proteins were transferred to a nitrocellulose membrane using a Trans-Blot System for $60 \mathrm{~min}$ at $300 \mathrm{~mA}$ (Bio-Rad, Hercules, CA, USA), and then washed twice in $1 \times$ Tris-buffered saline-Tween 20 (TBS$\mathrm{T}$ ), and blocked for $60 \mathrm{~min}$ at $37{ }^{\circ} \mathrm{C}$ in $5 \%$ Difco $^{\mathrm{TM}}$ Skim Milk (BD Biosciences, San Jose, CA, USA). The membrane was then incubated with anti-His-Tag monoclonal primary antibodies (1:1000; NovaGen, Madison, WI, USA) and anti- $\beta$-actin monoclonal primary antibodies (1:2000; ABGENT, Suzhou, China), with shaking overnight at $4{ }^{\circ} \mathrm{C}$. Thereafter, the membranes were washed using TBS-T, and incubated with horseradish peroxidase (HRP)-conjugated goat anti-mouse secondary antibody (1:2000; Bioworld, Shenzhen, China) in blocking buffer at $37{ }^{\circ} \mathrm{C}$ for $2 \mathrm{~h}$. The membranes were washed thoroughly using TBS-T, and the immunoreactive protein bands were visualized using Pierce ${ }^{\mathrm{TM}} \mathrm{ECL}$ Western Blotting Substrate, before imaging using the Universal Hood Gel Doc System (Bio-Rad).

\section{$\mathrm{C} 6 / 36$ cell viability assay}

The viability of cells overexpressing CYP325BB1 was investigated using a CCK-8 kit assay (Dojindo, Kumamoto, Japan) [26]. C6/36 cells $(100 \mu \mathrm{l})$ were added to each well of a 96-well plate at $5 \times 10^{3}$ cells/well and incubated in a $5 \% \mathrm{CO}_{2}$-humidified incubator at $28^{\circ} \mathrm{C}$ for $24 \mathrm{~h}$. At 24 $\mathrm{h}$ after transfection the cells were treated with $100 \mu \mathrm{l}$ of deltamethrin at concentrations of $0,10^{0.5}, 10^{1}, 10^{1.5}, 10^{2}$ and $10^{2.5} \mathrm{mg} / \mathrm{l}$ [27]. After a further $24 \mathrm{~h}$, the CCK- 8 solution $(10 \mu \mathrm{l})$ was added to each well and incubated at $28{ }^{\circ} \mathrm{C}$ for $3 \mathrm{~h}$. The absorbance was then measured using a dual wavelength spectrophotometer in a microplate reader at 450 and $630 \mathrm{~nm}$. Dimethyl sulfoxide (DMSO; Sigma, St. Louis, MO, USA) was used to dissolve deltamethrin at a final concentration of $0.5 \%(\mathrm{v} / \mathrm{v})$. Three biological replicates with three technical replicates were performed.

\section{Statistical analysis}

Statistically significant qualitative variables were detected using GraphPad Prism 8.0 software (GraphPad Software Inc., La Jolla, CA, USA). Data from independent experiments are presented as the mean \pm the standard error of mean (SEM). Student's t-test was used to determine the statistical significance of gene expression compared with that in the NC. The Chi-squared test $\left(\chi^{2}\right)$ was used to analyze mosquito mortality. Statistical significance was indicated by $P<0.05[28,29]$. All experiments were performed in at least three independent cohorts.

\section{Results \\ MiR-279-3p targets CYP325BB1}

Preliminary Solexa sequencing results showed that miR279-3p expression was significantly different between DR- and DS-strain mosquitoes [19]. Then, qRT-PCR was used to assess miR-279-3p expression in the DR- and DSstrains. MiR-279-3p expression was 2.97-fold higher in the DS-strain than in the DR-strain (Fig. $1 \mathrm{a} ;{ }^{* * *} P<0.001$ ). In contrast, the expression of $C Y P 325 B B 1$ was 2.31-fold higher in the DR-strain than in the DS-strain (Fig. 1b; *** $P<0.001)$. This contrasting expression pattern of miR279-3p and CYP325BB1 suggested that miR-279-3p might target $C Y P 325 B B 1$.

To verify this hypothesis, bioinformatic analysis was performed using the database of $C x$. quinquefasciatus P450 sequences, which showed that the $3^{\prime}$-UTR of CYP325BB1 harbored a miR-279-3p target site (Fig. 2a, b). To assess the regulatory relationship between the miRNA and the target mRNA, we performed a dual-luciferase reporter assay to detect the interaction between miR-279-3p and CYP325BB1 in vitro. The CYP325BB1 $3^{\prime}$-UTR-WT and $3^{\prime}$-UTR- $\Delta$ were inserted into the Renilla translational stop codon in the pMIR-REPORT miRNA 


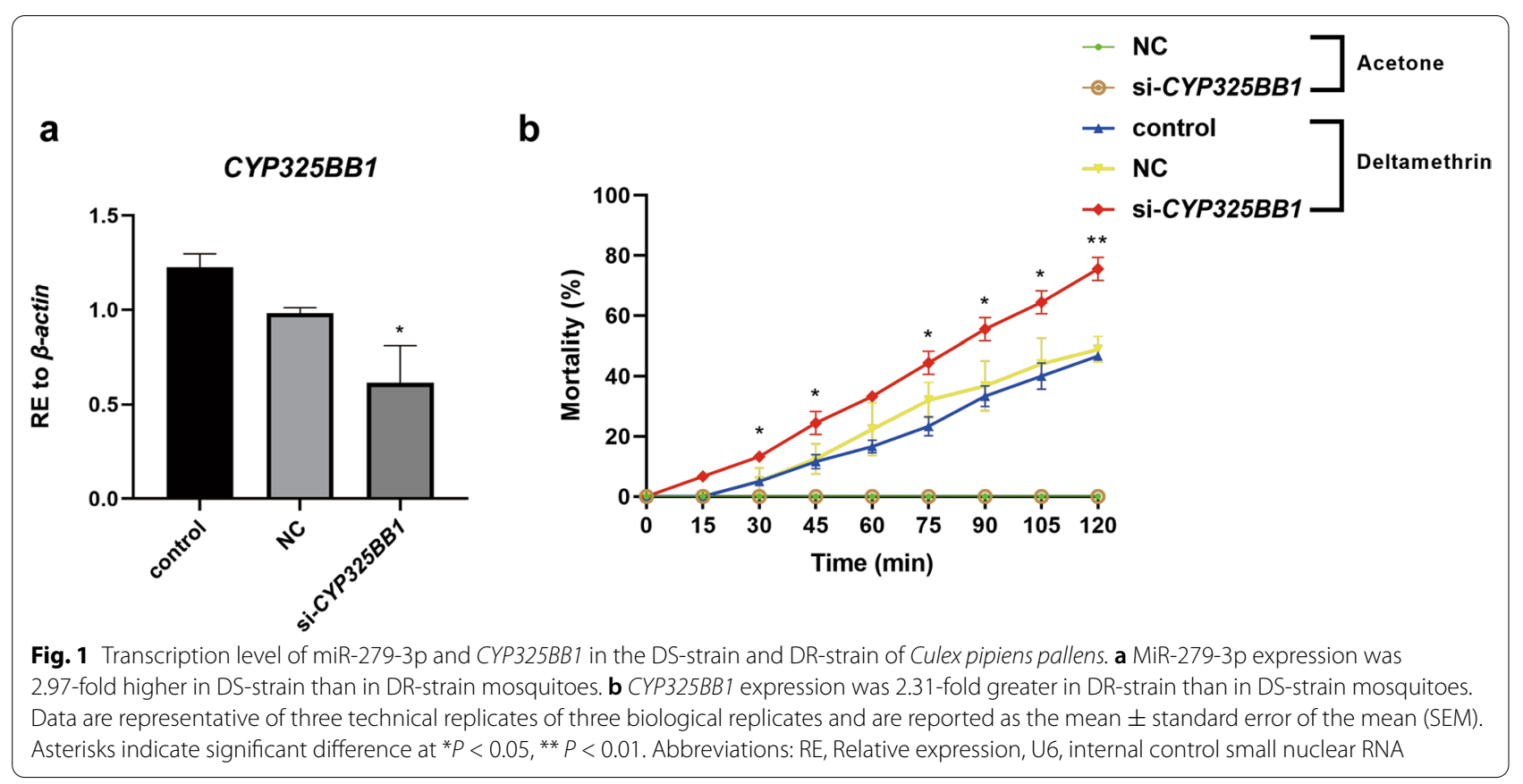

a

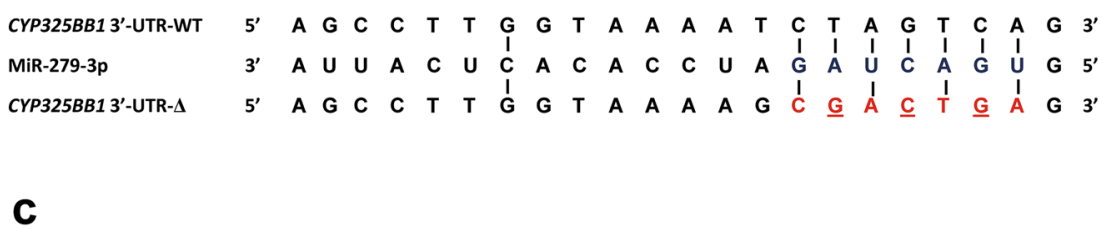

C

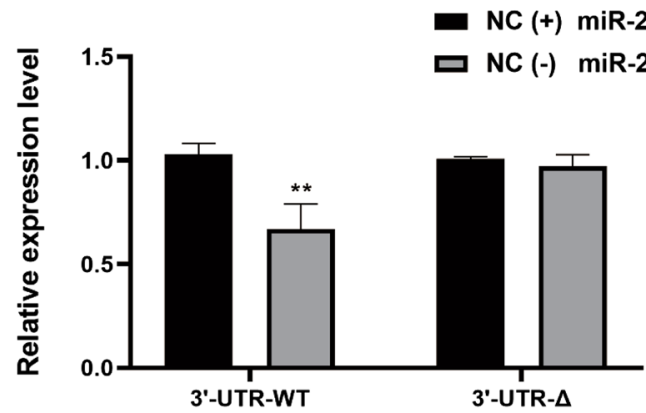

b

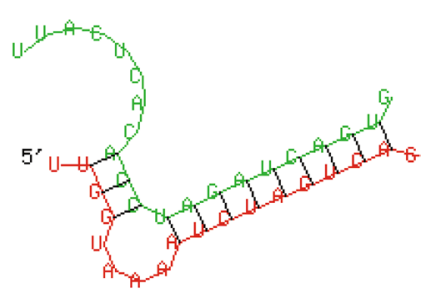

mfe: $-19.2 \mathrm{kcal} / \mathrm{mol}$

Fig. 2 MiR-279-3p targets CYP325BB1 via its 3'-UTR. a A region of the CYP325BB1 3'-UTR containing a putative binding site for miR-279-3p. b The free binding energy of CYP325BB1 targeted by miR-279-3p predicted by the RNAhybrid software. $\mathbf{c}$ A dual-luciferase reporter assay demonstrated 33.0\% decreased luciferase activity, suggesting that miR-279-3p could bind to the 3'-UTR of CYP325BB1. Data are representative of three technical replicates of three biological replicates and are reported as the mean \pm SEM. Asterisks indicate significant difference at ${ }^{* *} P<0.01$

Expression Reporter vector, generating $3^{\prime}-\mathrm{UTR}-\mathrm{WT} / \Delta$ fused luciferase reporters, which were cotransfected with control plasmid (PGL4.7) into HEK293T cells, and then treated with miRNA-279-3p mimic or NC. In the 3'-UTR-WT group, the luciferase intensity was inhibited by about $33.0 \%$, showing that miR-279-3p could bind to the $3^{\prime}$-UTR construct, while there was no significant change in luciferase intensity in the NC group. Importantly, there was no change in luciferase intensity in the $3^{\prime}$-UTR- $\Delta$ group, whether treated with the miR-279-3p mimic or NC (Fig. 2c; ** $P<0.01$ ). Therefore, CYP325BB1 was confirmed as a target gene of miR-279-3p in vitro. 


\section{MiR-279-3p modulates deltamethrin resistance} of mosquitoes

To validate the participation of miR-279-3p in the regulation of deltamethrin resistance, mosquitoes overexpressing miR-279-3p were constructed. The miR-279-3p mimic was injected into $12-\mathrm{h}$ PE DR-strain mosquitoes. The results showed that miR-279-3p was efficiently overexpressed (by 2.72-fold) in the miR-279-3p mimic injection group (Fig. 3a; ${ }^{* * * P} P<0.001$ ). To further validate the regulatory relationship between miR-279-3p and $C Y P 325 B B 1$ in vivo, the transcription level of CYP325BB1 was detected after overexpression of miR279-3p in mosquitoes. Overexpression of miR-279-3p downregulated the expression of CYP325BB1 by $61.3 \%$ (Fig. 3b; ${ }^{* *} P<0.01$ ). The results of the CDC bottle bioassay showed significantly higher mortality rates after the miR-279-3p mimic was injected compared with the control. After $105 \mathrm{~min}$, the experimental group showed a mortality rate of $61.7 \%$ (29/47), whereas the NC group showed a mortality rate of $43.9 \%(18 / 41)$; in comparison, the control group showed a mortality rate of $40.0 \%$ $(24 / 60)$. Furthermore, after $120 \mathrm{~min}$, the mortality rates were $74.5 \%(35 / 47)$ in the experimental group, $48.8 \%$ $(20 / 41)$ in the NC group, and $46.7 \%(28 / 60)$ in the control group (Fig. 3c; ${ }^{*} \mathrm{P}<0.05$ ).

For the biocontrol of mosquitoes, in vivo delivery through feeding is required; therefore, the designed miR-279-3p mimic was supplied orally to mosquitoes. After ingestion, the relative expression of miR-279-3p was 8.11-fold higher than that in the NC group, which suggested that miR-279-3p was successfully overexpressed in the DR-strain (Fig. 4a). Consistently, in the miR-279-3p mimic oral feeding group, the expression of $C Y P 325 B B 1$ was downregulated by $57.5 \%$ (Fig. 4b;
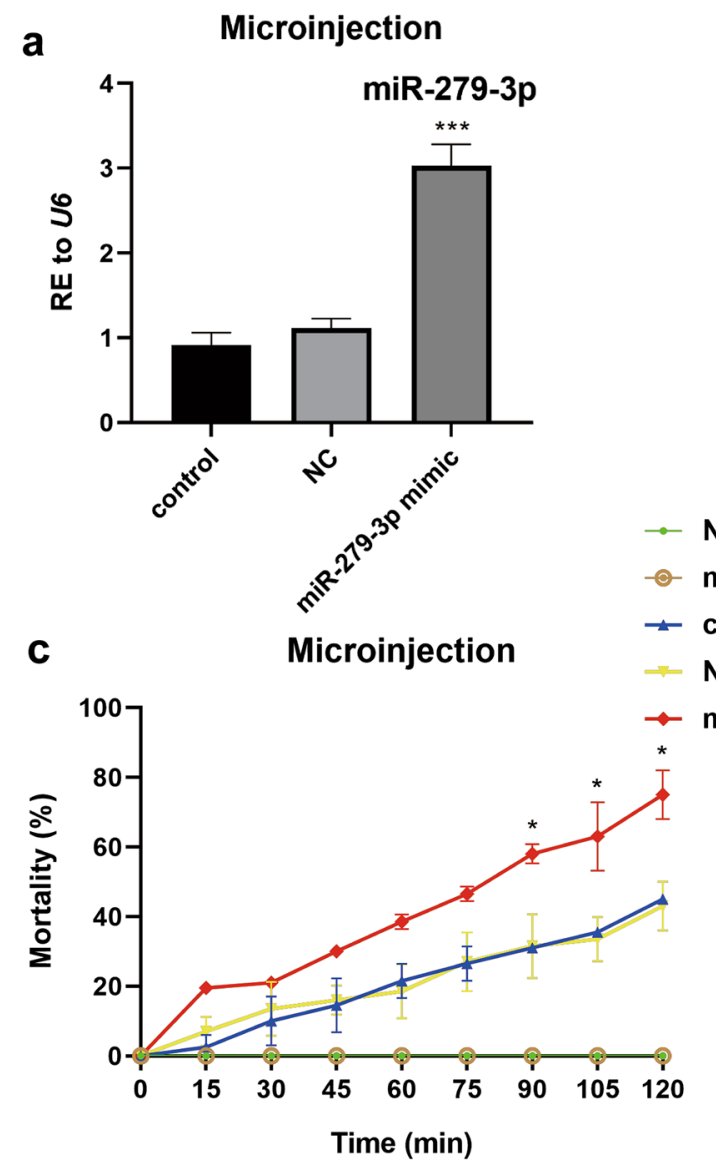

b

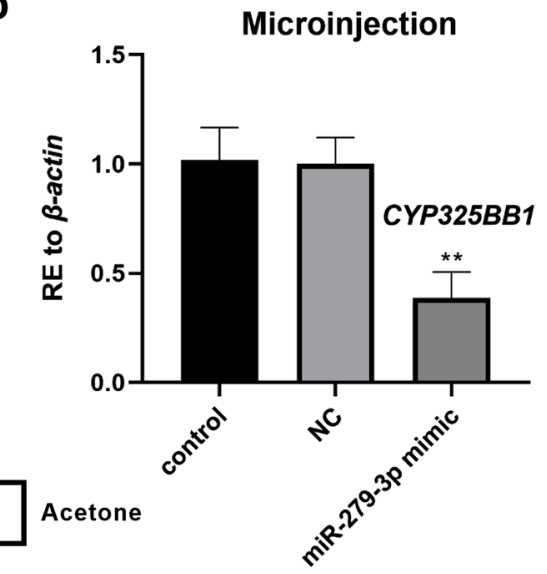

Deltamethrin

Fig. 3 MiR-279-3p-mimic-injected DR-strain mosquitoes are more sensitive to deltamethrin. a Relative expression of miR-279-3p was 2.72-fold higher in the DR-strain mosquitoes at $72 \mathrm{~h}$ after injection with the miR-279-3p mimic, compared to the NC and control groups. $\mathbf{b}$ Relative expression of CYP325BB1 was decreased by $61.3 \%$ in the DR-strain mosquitoes at $72 \mathrm{~h}$ after injection with the miR-279-3p mimic compared to the $\mathrm{NC}$ and control groups. c Mortality following $7 \mathrm{mg} / \mathrm{l}$ deltamethrin treatment of the miR-279-3p-mimic-injected group ranged from 57.4 to $74.5 \%$ at 90-120 min in the CDC bottle bioassay, which was higher than that in the acetone control, control and NC groups. Data are representative of three technical replicates of three biological replicates and are reported as the mean \pm SEM. Asterisks indicate significant difference at $* P<0.05, * * P<0.01$, *** $P<0.001$. Abbreviations: RE Relative expression 

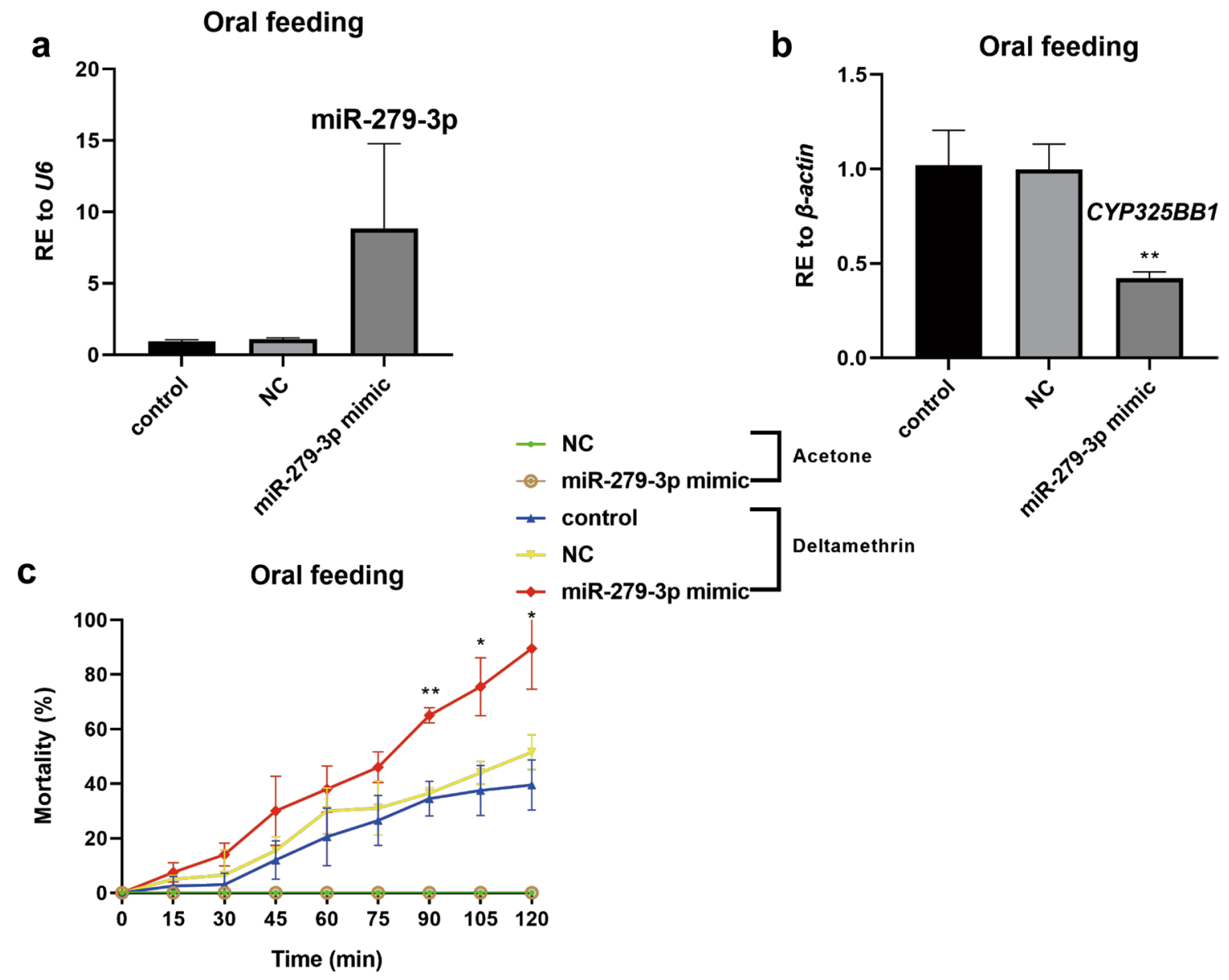

Fig. 4 MiR-279-3p-mimic-supplied DR-strain mosquitoes by oral feeding reduced the intensity of deltamethrin resistance. a Relative expression of miR-279-3p was upregulated by 8.11 -fold in the DR-strain mosquitoes at $48 \mathrm{~h}$ after oral feeding with the miR-279-3p mimic. $\mathbf{b}$ Relative expression of CYP325BB1 was decreased by $57.5 \%$ in the DR-strain mosquitoes at $48 \mathrm{~h}$ after oral feeding with the miR-279-3p mimic. c Mortality of the miR-279-3p mimic group after incubation in $7 \mathrm{mg} / \mathrm{l}$ deltamethrin-coated CDC bottles for $2 \mathrm{~h}$ was higher than that in the acetone control, control and NC groups. The mortality rate ranged from 64.9 to $89.2 \%$ in the miR-279-3p mimic group at 90-120 min. Data are representative of three technical replicates of three biological replicates and are reported as the mean \pm SEM. Statistical values are relative to the NC. Asterisks indicate significant difference at ${ }^{*} P<0.05,{ }^{*} P<0.01$. Abbreviations: RE, Relative expression

$\left.{ }^{* *} P<0.01\right)$. In the CDC bottle bioassay, the miR-279-3p mimic feeding group experienced higher mortality than did the NC and control groups. After $90 \mathrm{~min}$, the miR279-3p mimic group showed a mortality rate of $64.9 \%$ (24/37), whereas the NC group showed a mortality rate of $42.4 \%(14 / 33)$ and the control group showed a mortality rate of $33.3 \%$ (11/33). Furthermore, after $120 \mathrm{~min}$, the mortality rates were $89.2 \%(33 / 37), 57.6 \%(19 / 33)$ and $45.5 \%(15 / 33)$, respectively (Fig. 4 c; $* P<0.05$; $\left.{ }^{* *} P<0.01\right)$.

Microinjecting the DS-strain mosquitoes with the miR-279-3p inhibitor was then performed. The relative expression of miR-279-3p decreased by $40.2 \%$ compared with that in the NC1 group (Fig. $5 \mathrm{a}$; ${ }^{* *} P<0.01$ ) and, in contrast, the expression level of $C Y P 325 B B 1$ increased by 1.28 -fold compared with that in the $\mathrm{NC1}$ group (Fig. 5b; ${ }^{* *} P<0.01$ ). The results of the CDC bottle bioassay showed significantly lower mortality rates after injection of the miR-279-3p inhibitor compared with that of the control. After $105 \mathrm{~min}$, the experimental group showed a mortality rate of $14.9 \%$ (7/47), whereas the NC1 group showed a mortality rate of $40.0 \%$ $(18 / 45)$, and the control group showed a mortality rate of $43.1 \%(22 / 51)$. Furthermore, after $120 \mathrm{~min}$, the mortality rates were $21.3 \%(10 / 47)$ in the experimental group, $48.9 \%(22 / 45)$ in the NC1 group and $49.0 \%$ (25/51) in control group (Fig. 5 c; ${ }^{*} P<0.05$; ${ }^{* * * *} P<0.001$ ). These results suggested that $C Y P 325 B B 1$ is a bona fide target of miR-279-3p, that much higher expression of miR-279-3p is not intrinsically detrimental and that the 

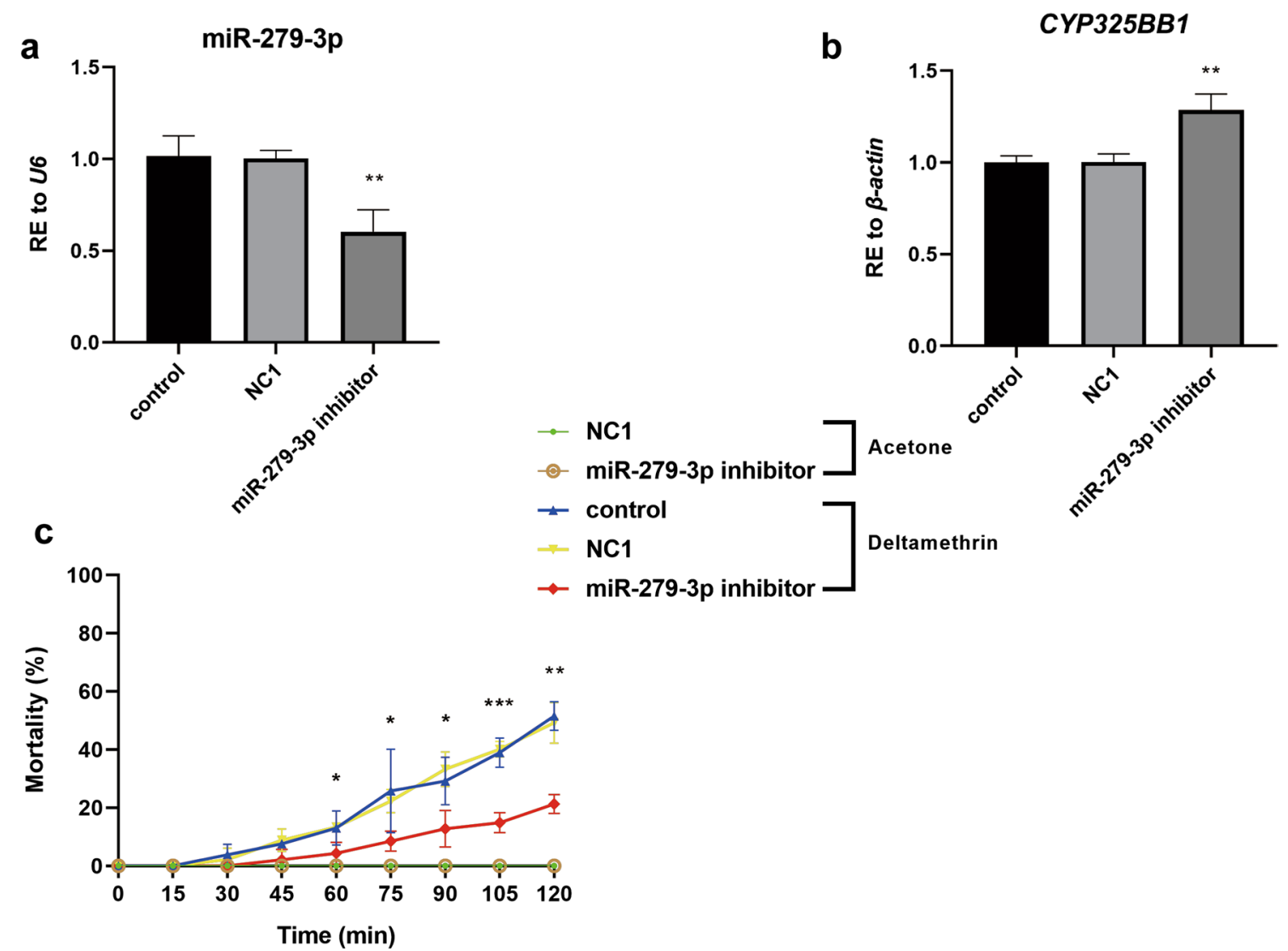

Fig. 5 MiR-279-3p-inhibitor-injected DS-strain mosquitoes are more resistant to deltamethrin. a Relative expression of miR-279-3p was decreased by $40.2 \%$ in the DS-strain mosquitoes at $72 \mathrm{~h}$ after injection with the miR-279-3p inhibitor. $\mathbf{b}$ The relative expression of CYP325BB1 was 1.28-fold higher in the DS-strain mosquitoes at $72 \mathrm{~h}$ after injection with the miR-279-3p inhibitor. c Mortality under $7 \mathrm{mg} / \mathrm{l}$ deltamethrin treatment of the miR-279-3p inhibitor-injected group ranged from 14.9 to $21.3 \%$ at 90-120 min in the CDC bottle bioassay, which was lower than that in the acetone control, control and NC1 groups. Data are representative of three technical replicates of biological replicates and are reported as the mean \pm SEM. Asterisks indicate significant difference at ${ }^{*} P<0.05,{ }^{* *} P<0.01,{ }^{* *} P<0.001$. Abbreviations: NC1, Inhibitor negative control 1; RE, relative expression

miR-279-3p could modulate deltamethrin resistance by targeting $C Y P 325 B B 1$ in mosquitoes.

\section{CYP325BB1 functions in the deltamethrin resistance \\ of mosquitoes}

The regulatory role of $C Y P 325 B B 1$ in mosquito resistance to deltamethrin in vitro was confirmed by amplifying the complete CYP325BB1 coding DNA sequence (GenBank: KM056313.1) from Cx. pipiens pallens and cloning it into the $\mathrm{pIB} / \mathrm{V} 5$-His vector to generate plasmid pIB/V5-His-CYP325BB1. Total RNA was extracted from $\mathrm{C} 6 / 36$ cells of the control group, the pIB/V5-His NC group and the experimental group (pIB/V5-His$C Y P 325 B B 1)$. The results showed that the expression level of $C Y P 325 B B 1$ was 397-fold higher than that in the NC group (Fig. 6a; ${ }^{* *} P<0.01$ ). Next, the protein expression level was validated using western blotting, and a band was detected using anti His-tag antibodies, which demonstrated that a protein of the expected size that was recognized by the anti-His-tag antibodies was expressed in the pIB/V5-His-CYP325BB1-transfected cells (Fig. 6b). Detection of the mRNA and protein levels proved that the transfection was successful. To investigate the sensitivity of the transiently transfected C6/36 cells to deltamethrin, a CCK-8 assay was employed to detect the viability of cells treated with deltamethrin in vitro. The proportion of viable cells was much higher among $\mathrm{pIB} /$ V5-His-CYP325BB1-transfected cells than cells in the $\mathrm{NC}$ group over a deltamethrin concentration range of $10^{1}-10^{2.5} \mathrm{mg} / \mathrm{l}$ (Fig. 6c; ${ }^{*} P<0.05$; ${ }^{* *} P<0.01$ ). Thus, the overexpression of $C Y P 325 B B 1$ could increase cell resistance to deltamethrin.

To further evaluate whether CYP325BB1 participates in mosquito resistance to deltamethrin in vivo, we conducted phenotypic observation experiments after CYP325BB1 RNAi knockdown (si-CYP325BB1) in DRstain mosquitoes. CYP325BB1 expression was $37.6 \%$ lower in the si-CYP325BB1 group than in the control 


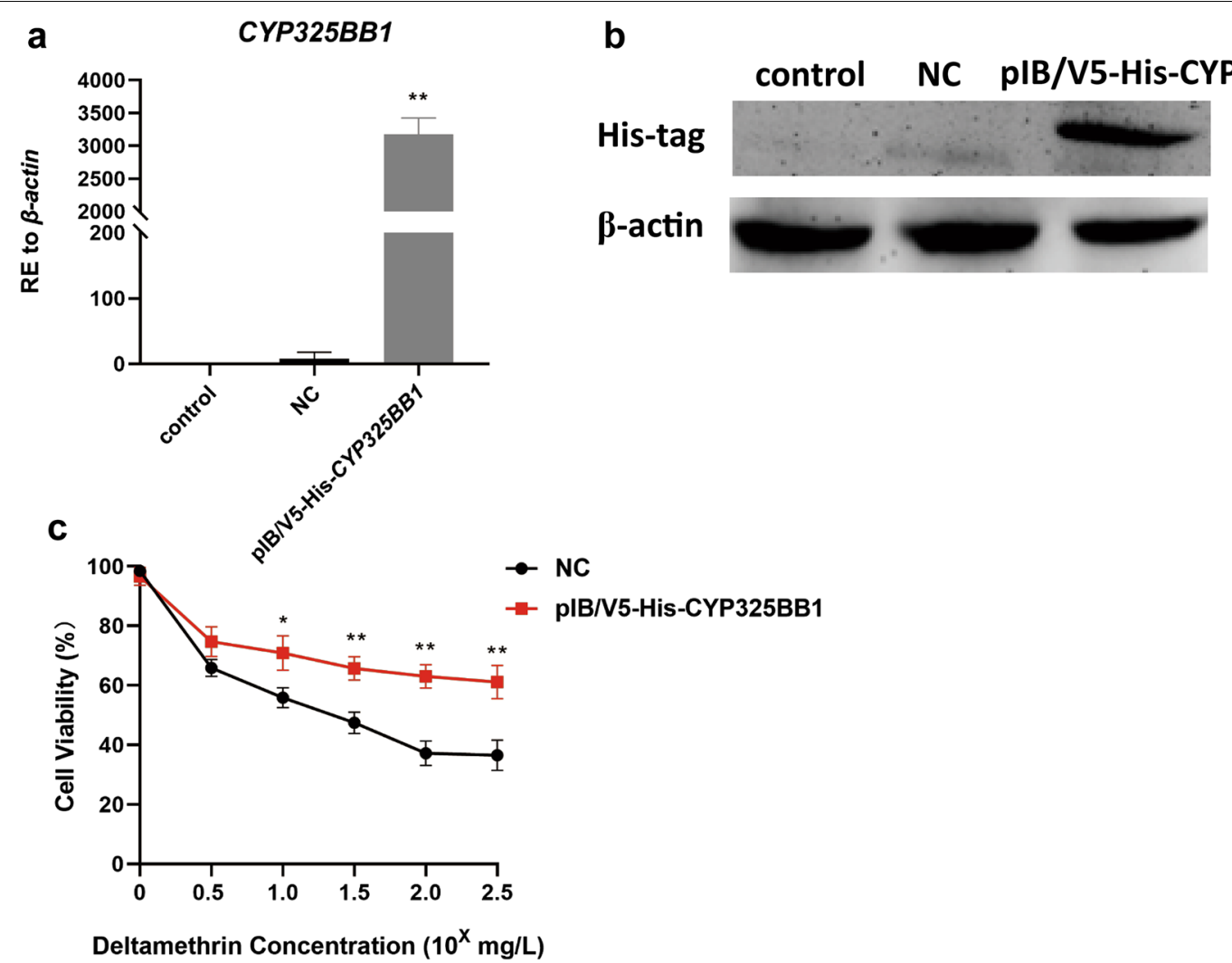

Fig. 6 The viability of deltamethrin-treated C6/36 cells was upregulated after overexpression of CYP325BB1 under stress. a Transcript level of CYP325BB1 in the pIB/N5-His-CYP325BB1-transfected C6/36 cells increased by 397-fold compared with that in the NC group. b His-tag antibody detection of the levels of the CYP325BB1 protein. $\mathbf{c}$ Results of the Cell Counting Kit-8 (CCK-8) showed that the viability of plB/ V5-His-CYP325BB1-transfected C6/36 cells treated with deltamethrin was higher than that in the plB/N5-His-transfected group. Data are representative of three technical replicates of biological replicates and are reported as the mean \pm SEM. Asterisks indicate a significant difference at ${ }^{*} P<0.05,{ }^{*} P<0.01$. Abbreviation: NC, plBN55-His negative control; RE, relative expression

and $\mathrm{NC}$ groups (Fig. 7a; $\left.{ }^{*} P<0.05\right)$. We then ran the CDC bottle bioassay to detect the mortality of si-CYP325BB1injected mosquitoes. From 30 to $120 \mathrm{~min}$, there was a significant difference in mortality, ranging from $13.3 \%$ $(6 / 45)$ to $75.6 \%(34 / 45)$ in si-CYP325BB1-injected mosquitoes compared with the control $(5.0 \%$ [3/60] to $46.7 \%$ [28/60]) and NC $(4.9 \%$ [2/41] to $48.8 \%$ [20/41]) groups (Fig. $7 \mathrm{~b} ; P<0.05,{ }^{* *} P<0.01$ ). Thus, RNAi silencing of CYP325BB1 in mosquitoes resulted in increased sensitivity to deltamethrin. Therefore, the RNAi of CYP325BB1 produced the same phenotypes that were caused by overexpression of the miR-279-3p mimic.

Taken together, these results indicated that miR279-3p might affect deltamethrin resistance by regulating CYP325BB1 in Cx. pipiens pallens.

\section{Discussion}

Mosquitoes and vector-borne diseases still represent a significant threat to human welfare; however, repeated exposures to insecticidal agents have caused high levels of resistance, representing an ongoing challenge to vector control [30]. Previous studies have provided evidence supporting the view that different mechanisms and genes are associated with the development of insecticide resistance [31]. MicroRNAs are short, well-characterized, noncoding, post-transcriptional gene regulators [32]. During the process of transforming primary precursors (primiRNAs) to pre-miRNAs, multiple regulatory proteins are involved that bind directly to distinct pre-miRNA in a sequence- or structure-dependent manner, to produce mature miRNAs [33]. Accordingly, miRNAs have been associated with a wide range of fundamental biological processes and are implicated in development, metabolism and host-pathogen interactions; indeed, many miRNAs are downregulated in the process of insecticide resistance [34]. In Drosophila melanogaster and Plutella xylostella (L.), changes in miRNA expression are induced by insecticides [35, 36]. Comparative and correlative studies have revealed that miRNAs are involved in the regulation of deltamethrin insecticide resistance; 


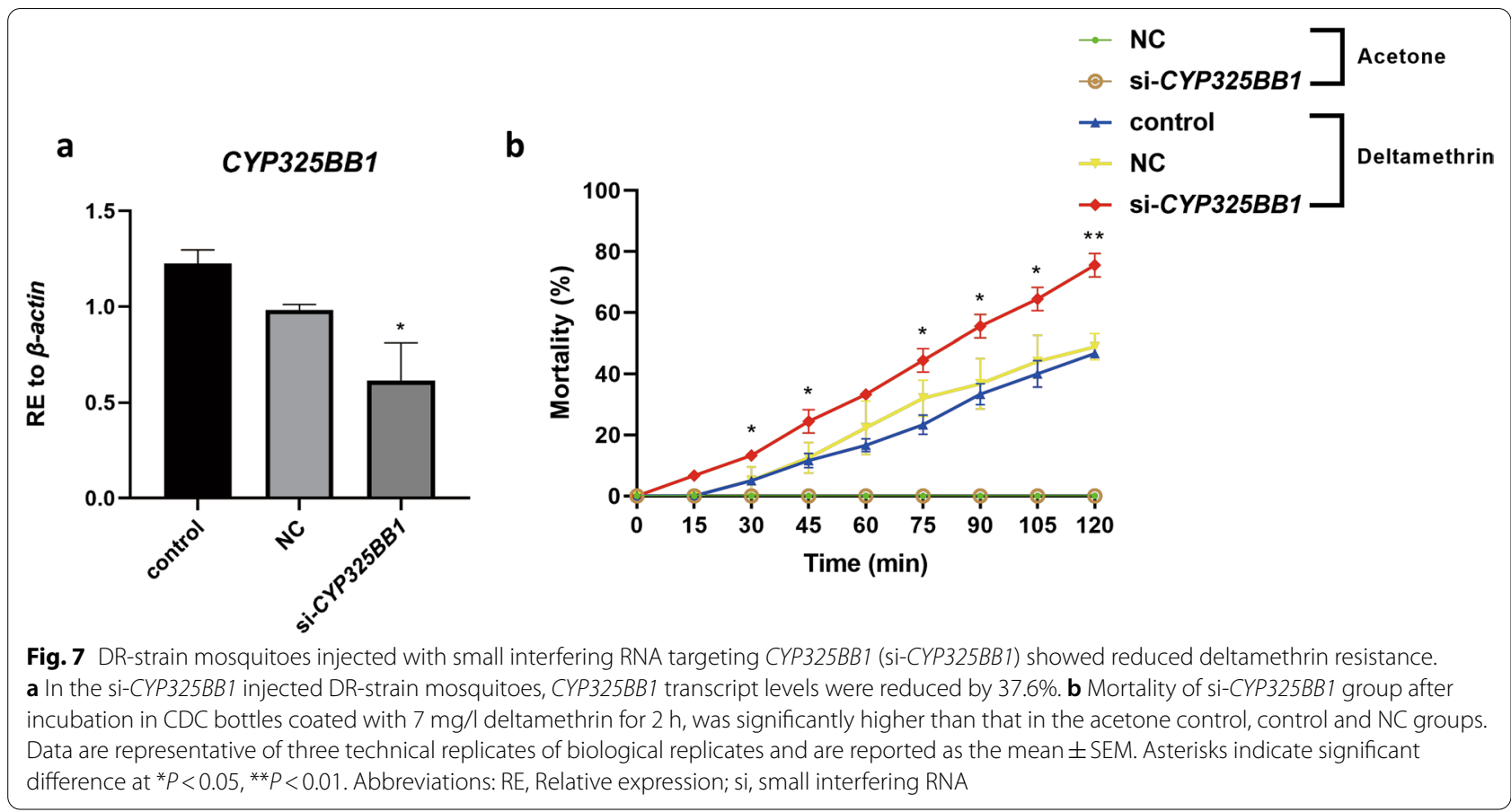

however, we lack strong evidence of miRNAs as causative factors of resistant phenotypes [14, 15, 37, 38].

Our previous high-throughput sequencing study identified miR-279-3p as being expressed at a significantly lower level in DR-strain mosquitoes compared to DSstrain mosquitoes [19]. The results of the present study demonstrated lower expression of miR-279-3p in the DR-strain compared with that in the DS-strain, which is consistent with the sequencing results. Certain miRNAs appear to show plasticity in transcription in the presence of pesticides in Cx. pipiens pallens, and certain miRNAs or miRNA clusters are downregulated in the DR-strain, such as miR-2 13 17, miR-4448 and miR-278-3p [14, $16,17]$. However, other miRNAs are upregulated in the DR-strain, such as miRNA-92a, miRNA-932 and miR$285[15,38,39]$.

The miRNA we investigated is constitutively downregulated in the DR-strain. The miR-279-3p-mimicinjected DR-strain mosquitoes became more susceptible to deltamethrin, while the miR-279-3p-inhibitor-injected DS-strain mosquitoes became more resistant to deltamethrin, which validated that miR-279-3p participates in the regulation of deltamethrin resistance. The convenient, effective and environmentally friendly use of the miR279-3p mimic as a molecular biopesticide in the future requires would require its oral feeding [40]. Therefore, the miR-279-3p mimic was delivered orally to induce RNA interference in the DR-strain mosquitoes. The mortality was higher in the miR-279-3p-mimic-fed group than in the NC group, which further confirmed miR-279$3 p$-induced regulation of mosquito resistance. According to previous reports, E. coli and Saccharomyces cerevisiae expression systems could be used for silencing target genes. The bioinsecticide could be used as an insecticidal adjuvant agent, thereby minimizing the environmental impact. However, further studies are necessary to establish the conditions for expression and large-scale culture, and to determine the effectiveness of this bioinsecticide via different forms of administration, such as spraying, in all stages of vector development. In addition, the use of this expression system in field applications should be regulated by the appropriate authorities in each country $[41,42]$.

MiRNA genes are encoded within the genome. Their transcription might be tightly coordinated with the transcription of other genes that serve either as a source of miRNAs or as their targets, including the protein-coding genes to exert miRNA biogenesis functions [43]. In support of this model, intronic miR-208a, which is coexpressed with its heart-specific host gene, Myh6, controls the suppression of negative regulators of muscle growth and hypertrophy in mice [44]. Recent studies on miRNA-mediated metabolic insecticide resistance have revealed that aberrant expression of miRNAs can induce marked changes in the expression levels of detoxification genes, especially those encoding cytochrome P450s [14, 37, 45]. Therefore, we firstly identified CYP325BB1 as the miR-279-3p's 
direct target in vitro and in vivo. In the dual-luciferase reporter assay, cotransfection of the luciferase reporter vector comprising the CYP325BB1 3 '-UTR together with the miR-279-3p mimic caused a decrease in Renilla luciferase activity in vitro, and the overexpression of miR-279-3p reduced the CYP325BB1 expression level in vivo. Taken together, these results confirmed CYP325BB1 as an authentic target of miR-279-3p in mosquitoes.

Studies have demonstrated that increased expression of cytochrome P450s could enhance metabolic detoxification of insecticides in resistant mosquitoes [46, 47]. Thus, we investigated the mechanism of CYP325BB1"s contribution to insecticide resistance following the functional test of $C Y P 325 B B 1$ in vitro and in vivo. The CCK-8 assay was used to detect C6/36 cell viability in vitro because $C Y P 325 B B 1$ expression showed that cell viability was higher, indicating that this cytochrome is involved in deltamethrin resistance. Additionally, we injected si-CYP325BB1 into mosquitoes and detected deltamethrin resistance in vivo. Our results showed higher mortality in the CYP325BB1-silenced group, indicating that reduced CYP325BB1 expression abrogated deltamethrin resistance.

The findings of the present study demonstrate a link between decreased miR-279-3p expression and a deltamethrin resistance phenotype via post-transcriptional suppression of $C Y P 325 B B 1$, whose protein product is involved in xenobiotic detoxification. Therefore, miR279-3p could represent a novel biomarker of insecticide exposure, and is one of the factors that could explain the insecticide's toxic effects. These insights are important to understand how mosquitoes have adapted at the molecular level to evolve deltamethrin resistance, and highlight the importance of gaining a complete understanding of insect biology for insecticide studies.

\section{Abbreviations \\ CDC: U.S. Centers for Disease Control and Prevention; CYP: Cytochrome P450; DR-strain: Deltamethrin-resistant strain; DS-strain: Deltamethrin-sensitive strain; miRs: MicroRNAs; NC: Negative control; qRT-PCR: Quantitative real-time reverse transcription PCR; UTR: Untranslated region; WT: Wild-type.}

\section{Acknowledgements}

Not applicable.

\section{Authors' contributions}

XL: Conceptualization, methodology, data curation, software, rormal analysis, validation, writing-review and editing. SH: Conceptualization, methodology, data curation, software, formal analysis, writing-original draft preparation. $\mathrm{HZ}$ : Validation, revision-review, editing; HY: Data curation, validation. HW: Data curation. YS: Funding acquisition, supervision. DZ: Funding acquisition, supervision. LM: Resources, supervision. BS: Funding acquisition, project administration, supervision. Changliang Zhu: Project administration, supervision. All authors read and approved the final manuscript.

\section{Funding}

This work was funded by the National Natural Science Foundation of China (Grant Nos. 81672058, 81672056, 81772227, and 81971970) and the National S \& T Major Program (Grant No. 2017ZX10303404-002-006).

\section{Availability of data and materials}

All data generated or analyzed during this study are included in this published article.

\section{Declarations}

\section{Ethics approval and consent to participate}

Procedures for blood-feeding with mice in our laboratory were approved by The National Science and Technology of China and People's Government of Jiangsu Province Animal Care and Use Committee and Institutional Review Board (No. IACUC-1812047).

\section{Consent for publication}

Not applicable.

\section{Competing interests}

The authors declare that they have no competing interests.

\section{Author details}

${ }^{1}$ Department of Pathogen Biology, Nanjing Medical University, Nanjing, Jiangsu 211166, People's Republic of China. '2Department of Pathogen Biology, Nanjing University of Chinese Medicine, Nanjing, Jiangsu 210046, People's Republic of China. ${ }^{3}$ Department of Stomatology, Fifty People's Hospital of Yuhang District, Hangzhou, Zhejiang 311199, People's Republic of China.

Received: 28 May 2021 Accepted: 23 September 2021

Published online: 12 October 2021

\section{References}

1. Achee NL, Grieco JP, Vatandoost H, Seixas G, Pinto J, Ching-Ng L, et al. Alternative strategies for mosquito-borne arbovirus control. PLoS Neglect Trop Dis. 2019;13(1):e0006822.

2. Manet C, Roth C, Tawfik A, Cantaert T, Sakuntabhai A, Montagutelli X. Host genetic control of mosquito-borne Flavivirus infections. Mamm Genome. 2018;29(7-8):384-407.

3. Global Malaria Programme. WHO Guidelines for malaria. WHO/UCN/ GMP/2021.01 Rev.1.2021.07.

4. Ashley EA, Pyae Phyo A, Woodrow CJ. Malaria. Lancet. 2018;391(10130):1608-21.

5. Alout H, Labbe P, Chandre F, Cohuet A. Malaria vector control still matters despite insecticide resistance. Trends Parasitol. 2017;33(8):610-8.

6. Barbosa S, Kay K, Chitnis N, Hastings IM. Modelling the impact of insecticide-based control interventions on the evolution of insecticide resistance and disease transmission. Parasit Vectors. 2018;11(1):482.

7. Liu N. Insecticide resistance in mosquitoes: impact, mechanisms, and research directions. Annu Rev Entomol. 2015;60:537-59.

8. Scott JG. Cytochromes P450 and insecticide resistance. Insect Biochem Mol Biol. 1999;29(9):757-77.

9. Huang Y, Guo Q, Sun X, Zhang C, Xu N, Xu Y, et al. Culex pipiens pallens cuticular protein CPLCG5 participates in pyrethroid resistance by forming a rigid matrix. Parasit Vectors. 2018;11(1):6.

10. Bartel DP. MicroRNAs: genomics, biogenesis, mechanism, and function. Cell. 2004;116(2):281-97.

11. Wang JX, Gao J, Ding SL, Wang K, Jiao JQ, Wang Y, et al. Oxidative modification of miR-184 enables it to target Bcl-xL and Bcl-w. Mol Cell. 2015;59(1):50-61.

12. Liu S, Lucas KJ, Roy S, Ha J, Raikhel AS. Mosquito-specific microRNA-1174 targets serine hydroxymethyltransferase to control key functions in the gut. Proc Natl Acad Sci USA. 2014;111(40):14460-5.

13. Lampe $L$, Jentzsch M, Kierszniowska S, Levashina EA. Metabolic balancing by miR-276 shapes the mosquito reproductive cycle and Plasmodium falciparum development. Nat Commun. 2019;10(1):5634. 
14. Guo Q, Huang Y, Zou F, Liu B, Tian M, Ye W, et al. The role of miR-2 approximately 13 approximately 71 cluster in resistance to deltamethrin in Culex pipiens pallens. Insect Biochem Mol Biol. 2017;84:15-22.

15. Liu B, Tian M, Guo Q, Ma L, Zhou D, Shen B, et al. MiR-932 Regulates pyrethroid resistance in Culex pipiens pallens (Diptera: Culicidae). J Med Entomol. 2016;53(5):1205-10.

16. Lei Z, Lv Y, Wang W, Guo Q, Zou F, Hu S, et al. MiR-278-3p regulates pyrethroid resistance in Culex pipiens pallens. Parasitol Res. 2015:114(2):699-706.

17. Li X, Hu S, Yin H, Zhang H, Zhou D, Sun Y, et al. MiR-4448 is involved in deltamethrin resistance by targeting CYP4H31 in Culex pipiens pallens. Parasit Vectors. 2021;14(1):159.

18. Feng $X$, Zhou S, Wang J, Hu W. microRNA profiles and functions in mosquitoes. PLoS Neglect Trop Dis. 2018;12(5):e0006463.

19. Hong S, Guo Q, Wang W, Hu S, Fang F, Lv Y, et al. Identification of differentially expressed microRNAs in Culex pipiens and their potential roles in pyrethroid resistance. Insect Biochem Mol Biol. 2014;55:39-50.

20. Dedeoglu BG. High-throughput approaches for microRNA expression analysis. Methods Mol Biol. 2014;1107:91-103.

21. Yu X, Zhou Q, Li SC, Luo Q, Cai Y, Lin WC, et al. The silkworm (Bombyx mori) microRNAs and their expressions in multiple developmental stages. PLoS ONE. 2008;3(8):e2997.

22. Omondi BA, Latorre-Estivalis JM, Rocha Oliveira $I H$, Ignell $R$, Lorenzo MG. Evaluation of reference genes for insect olfaction studies. Parasit Vectors. 2015;8:243.

23. Livak KJ, Schmittgen TD. Analysis of relative gene expression data using real-time quantitative PCR and the 2(- Delta Delta $C(T))$ Method. Methods. 2001;25(4):402-8

24. Rehmsmeier M, Steffen P, Hochsmann M, Giegerich R. Fast and effective prediction of microRNA/target duplexes. RNA. 2004;10(10):1507-17.

25. William G. Brogdon, Adeline Chan. Guideline for Evaluating Insecticide Resistance in Vectors Using the CDCBottle Bioassay. https://www.cdc. gov/malaria/resources/pdf/fsp/ir_manual/ir_cdc_bioassay_en.pdf. 2015;11.

26. Yu J, Hu S, Ma K, Sun L, Hu H, Zou F, et al. Ribosomal protein S29 regulates metabolic insecticide resistance through binding and degradation of CYP6N3. PLOS ONE. 2014;9(4):e94611.

27. Sun H, Sun L, He J, Shen B, Yu J, Chen C, et al. Cloning and characterization of ribosomal protein $\$ 29$, a deltamethrin resistance associated gene from Culex pipiens pallens. Parasitol Res. 2011:109(6):1689-97.

28. Zou FF, Guo Q, Sun Y, Zhou D, Hu MX, Hu HX, et al. Identification of protease $\mathrm{m} 1$ zinc metalloprotease conferring resistance to deltamethrin by characterization of an AFLP marker in Culex pipiens pallens. Parasit Vectors. 2016;9:172.

29. Dagg K, Irish S, Wiegand RE, Shililu J, Yewhalaw D, Messenger LA. Evaluation of toxicity of clothianidin (neonicotinoid) and chlorfenapyr (pyrrole) insecticides and cross-resistance to other public health insecticides in Anopheles arabiensis from Ethiopia. Malar J. 2019;18(1):49.

30. Wilson AL, Courtenay O, Kelly-Hope LA, Scott TW, Takken W, Torr SJ, et al. The importance of vector control for the control and elimination of vector-borne diseases. PLoS Neglect Trop Dis. 2020;14(1):e0007831.

31. Auteri M, La Russa F, Blanda V, Torina A. Insecticide resistance associated with kdr mutations in Aedes albopictus: an update on worldwide evidences. Biomed Res Int. 2018;2018:3098575.
32. Ullah M, Ng NN, Concepcion W, Thakor AS. Emerging role of stem cellderived extracellular microRNAs in age-associated human diseases and in different therapies of longevity. Ageing Res Rev. 2020;57:100979.

33. Urbanek-Trzeciak MO, Jaworska E, Krzyzosiak WJ. miRNAmotif-a tool for the prediction of Pre-miRNA(-)protein interactions. Int J Mol Sci. 2018;19(12):4075

34. Aguilar C, Mano M, Eulalio A. Multifaceted roles of microRNAs in hostbacterial pathogen interaction. Microbiol Spectr. 2019;7(3). https://doi. org/10.1128/microbiolspec.BAI-0002-2019

35. Seong KM, Coates BS, Pittendrigh BR. Post-transcriptional modulation of cytochrome P450s, Cyp6g1 and Cyp6g2, by miR-310s cluster is associated with DDT-resistant Drosophila melanogaster strain 91-R. Sci Rep. 2020;10(1):14394

36. Li X, Guo L, Zhou X, Gao X, Liang P. miRNAs regulated overexpression of ryanodine receptor is involved in chlorantraniliprole resistance in Plutella xylostella (L.). Sci Rep. 2015:5:14095.

37. Sun $X H, X u N, X u Y$, Zhou D, Sun Y, Wang WJ, et al. A novel miRNA, miR13664, targets CpCYP314A1 to regulate deltamethrin resistance in Culex pipiens pallens. Parasitology. 2019;146(2):197-205.

38. Tian M, Liu B, Hu H, Li X, Guo Q, Zou F, et al. MiR-285 targets P450 (CYP6N23) to regulate pyrethroid resistance in Culex pipiens pallens. Parasitol Res. 2016;115(12):4511-7.

39. Ma K, Li X, Hu H, Zhou D, Sun Y, Ma L, et al. Pyrethroid-resistance is modulated by miR-92a by targeting CpCPR4 in Culexpipiens pallens. Comp Biochem Physiol B Biochem Mol Biol. 2017;203:20-4.

40. Ghosh SKB, Hunter WB, Park AL, Gundersen-Rindal DE. Double-stranded RNA oral delivery methods to induce RNA interference in phloem and plant-sap-feeding Hemipteran insects. J Vis Exp. 2018(135).

41. Mysore K, Hapairai LK, Sun L, Harper El, Chen Y, Eggleson KK, et al. Yeast interfering RNA larvicides targeting neural genes induce high rates of Anopheles larval mortality. Malar J. 2017;16(1):461.

42. Lopez SBG, Guimaraes-Ribeiro V, Rodriguez JVG, Dorand F, Salles TS, Sa-Guimaraes TE, et al. RNAi-based bioinsecticide for Aedes mosquito control. Sci Rep. 2019;9(1):4038

43. O'Carroll D, Schaefer A. General principals of miRNA biogenesis and regulation in the brain. Neuropsychopharmacol Off Publ Am Coll Neuropsychopharmacol. 2013;38(1):39-54.

44. Callis TE, Pandya $K$, Seok HY, Tang RH, Tatsuguchi M, Huang ZP, et al. MicroRNA-208a is a regulator of cardiac hypertrophy and conduction in mice. J Clin Investig. 2009;119(9):2772-86.

45. Qiao J, Du Y, Yu J, Guo J. MicroRNAs as potential biomarkers of insecticide exposure: a review. Chem Res Toxicol. 2019:32(11):2169-81.

46. Vontas J, Katsavou E, Mavridis K. Cytochrome P450-based metabolic insecticide resistance in Anopheles and Aedes mosquito vectors: muddying the waters. Pesticide Biochem Physiol. 2020;170:104666.

47. Liu N, Li M, Gong Y, Liu F, Li T. Cytochrome P450s—their expression, regulation, and role in insecticide resistance. Pestic Biochem Physiol. 2015;120:77-81.

\section{Publisher's Note}

Springer Nature remains neutral with regard to jurisdictional claims in published maps and institutional affiliations.

Ready to submit your research? Choose BMC and benefit from

- fast, convenient online submission

- thorough peer review by experienced researchers in your field

- rapid publication on acceptance

- support for research data, including large and complex data types

- gold Open Access which fosters wider collaboration and increased citations

- maximum visibility for your research: over 100M website views per year

At BMC, research is always in progress.

Learn more biomedcentral.com/submissions 